\title{
A note on the dimension of isometry group of a Riemannian manifold
}

\author{
Hulya Kadioglu \\ Yildiz Technical University, Istanbul, Turkey \\ Received: 9 August 2016, Accepted: 27 February 2017 \\ Published online: 12 August 2017.
}

\begin{abstract}
In this paper, we obtain some results on the dimension of the isometry group of a Riemannian manifold. In specific dimensions, we give a range which the dimension of an isometry group can not be in. We also give necessary conditions for a manifold to have a free canonical action on some specific manifolds. We give a boundary of the dimension of the full isometry group if the dimension of a manifold is greater or equal to 4 .
\end{abstract}

Keywords: Isometry groups of riemannian manifolds, noncompact lie groups of transformations, manifolds of metrics.

\section{Introduction}

It is well known that the group of isometries of a Riemannian manifold carries a Lie group structure acting on $\mathfrak{M}$ as a Lie transformation group. Therefore an isometry group of a Riemannian manifold has both algebraic and smooth structures. Its manifold topology is the compact-open topology which was introduced by Ralph Fox in 1945 [2]. This group is compact if the manifold is compact.

On the other hand the boundaries of the dimension of isometry groups has long been studied by researchers. If $M$ is an $n$ - dimensional Riemannian manifold and $G$ is a closed subgroup of $I(M)$, the group of isometries of $M$, it is a classical result that $\operatorname{dim} G<\frac{1}{2} n(n+1)$. H. C. Wang [11], and H. Wakakuwa [10] gives some other results on the dimension of the isometry group. Moreover Ihrig [3] proved that under suitable smoothness conditions, the upper bound is $\frac{n(n+1)}{2}$ if $\mathfrak{M}$ is an $n$-dimensional manifold which is homeomorphic to a topological metric space.

In this paper, we give some other boundaries if the manifold $\mathfrak{M}$ or its canonical action carries certain conditions. If $\operatorname{Dim}(\mathfrak{M}) \geq 4$, then the upper bound is $\frac{n(n-1)}{2}+1$ if $\mathfrak{M}$ is not of constant curvature. We also prove that if $\mathfrak{M}$ is an $n$-dimensional Riemannian manifold, where $\mathfrak{M}$ is isometric to one of the elements of $\mathfrak{A}=\left\{S^{n}, \mathbb{R}^{n}, P_{n}(\mathbb{R}), \mathbb{H}^{n}\right\}$ of constant curvature, and $(\mathfrak{E}, \pi, \mathfrak{M}, E)$ be a vector bundle, then if the canonical isometric action on $\mathfrak{M}$ is free , there is a following relation between $\operatorname{Dim}(\mathfrak{M})=n$ and $\operatorname{Dim}(E)=k$,

$$
k=\frac{t(t-1)}{2} \Longleftrightarrow n \in\{1,2, \ldots, t\}
$$

where $t=2,3, \ldots$.

In this study, we assume that all manifolds are smooth, second countable, and Hausdorff.

* Corresponding author e-mail: hkadio@yildiz.edu.tr 


\section{Preliminaries}

In this section, we give some preliminary information that we use throughout this paper. First we define induced metric on the total space of a vector bundle.

Let $\pi: \mathfrak{E} \rightarrow \mathfrak{M}$ be a vector bundle, where $(\mathfrak{M}, \mathfrak{g})$ is an $n$-dimensional Riemannian manifold, and suppose that $(\mathfrak{U}, E, \Phi)$ is a local fiber bundle trivialization of $\mathfrak{E}$ with $h \in \pi^{-1}(\mathfrak{U})$ and identify $T E$ with $E \times E$. It was proven in [4] that there exists an induced Riemannian metric $\tilde{\mathfrak{g}}$ on $\mathfrak{E}$ as follows.

$$
\tilde{\mathfrak{g}}\left(V_{h}, W_{h}\right)=\mathfrak{g}\left((\pi)_{*}(V),(\pi)_{*}(W)\right)+Q\left(\left(p r_{2} \circ \Phi\right)_{*}(V),\left(p r_{2} \circ \Phi\right)_{*}(W)\right)
$$

where $Q\left((e, v),\left(e^{\prime}, v^{\prime}\right)\right)=<e, e^{\prime}>+<v, v^{\prime}>$ for all $(e, v),\left(e^{\prime}, v^{\prime}\right) \in T E$.

In [5], it was proven in [5] that using the above metric, one can define a one to one Lie group homomorphism between isometry groups of base and total space of the vector bundle. The following theorem takes care of this issue.

Theorem 1. [5] Let $I(\mathfrak{M})$ and $I(\mathfrak{E})$ denote isometry groups of $\mathfrak{M}$ and $\mathfrak{E}$ respectively. Suppose that for each $x \in \mathfrak{M}$ we fix a local trivialization $\Phi$ around $x$, and denote it as $\Phi_{x}$ (similarly, when we pick another point $y \in \mathfrak{M}$, the fixed trivialization will be $\left.\Phi_{y}\right)$. Therefore when we pick a point $x \in \mathfrak{M}$, then $\Phi_{x}$ is uniquely defined. Let $f \in I(\mathfrak{M})$. Then the function

$$
\begin{aligned}
\Omega: I(\mathfrak{M}) & \rightarrow I(\mathfrak{E}) \\
\quad f & \rightarrow \quad \Omega(f)=\Phi_{f(x)}^{-1}\left(f \circ \pi, p r_{2} \circ \Phi_{x}\right)
\end{aligned}
$$

is a Lie group homomorphism.

It was also proven that the image of this homomorphism is a closed subgroup of the isometry group of $\mathfrak{E}$. Now, let $G=I(\mathfrak{M})$ and the image $\operatorname{Im}(\Omega)=\widetilde{G}$.

Theorem 2. [5] Let $\tilde{G}$ denotes the image of the function $\Omega$. Then

$$
\widetilde{G}=\left\{F \in I(\mathfrak{E}) \mid F=\Phi_{f(x)}^{-1}\left(f \circ \pi, p r_{2} \circ \Phi_{x}\right), f \in G\right\}
$$

is a Lie subgroup of $I(\mathfrak{E})$.

The subgroup $\widetilde{G}$ carries the relative topology to $I(\mathfrak{E})$. This makes the restriction of the function $\Omega$ ( the restriction to its image) a Lie group isomorphism.

Corollary 1.[5] The function $\left.\Omega\right|_{\Omega^{-1}(\widetilde{G})}$ is a Lie group isomorphism. Thus $\widetilde{G}$ is of dimension $N$, where Dim $(I(\mathfrak{M}))=N$.

We use the above corollary to decide the dimension of the isometry group of $\mathfrak{M}$ in certain conditions.

Theorem 3. [8] Let $\mathfrak{M}$ be an n-dimensional Riemannian manifold with $n \neq 4,6,10$. Then the group I( $\mathfrak{M})$ of isometries contains no closed subgroup $G$ where the dimension of $G$ falls into any of the ranges:

$$
\frac{1}{2}(n-k)(n-k+1)+\frac{1}{2} k(k+1)<\operatorname{dim} G<\frac{1}{2}(n-k+1)(n-k+2), \quad k=1,2,3, \ldots
$$

\section{Theory}

It is well known that the dimension of an isometry group of a Riemannian manifold is at most $\frac{n(n+1)}{2}$. In this section, we give some boundaries to the dimension of isometry groups by using the structures defined in previous sections. First we define the induced action on the total space of a vector bundle. 
Definition 1. Let $\mu$ be the canonical action of $I(\mathfrak{M})$ on $M$. Then the canonical $\tilde{G}$ action on $\mathfrak{E}$ is defined in a usual way as follows.

$$
\tilde{\mu}(F, h)=F(h),
$$

where $F \in \widetilde{G}$. We will call this action as the induced action on $\mathfrak{E}$.

Theorem 4. Let $(\mathfrak{E}, \pi, \mathfrak{M})$ is a vector bundle, in which the total space of the bundle endows with the induced metric $g_{\mathfrak{E}}$. Then the following properties hold

(i) If $I(\mathfrak{M})$ acts freely on $\mathfrak{M}$, then $\widetilde{G}$ acts freely on $\mathfrak{E}$.

(ii) If $\operatorname{dim}(\mathfrak{E}) \neq 4,6,10$, then the dimension of isometry group $I(\mathfrak{M})$ does not fall into any of the ranges

$$
\frac{1}{2}(n-k)(n-k+1)+\frac{1}{2} k(k+1)<\operatorname{dim}(I(\mathfrak{M}))<\frac{1}{2}(n-k+1)(n-k+2), \quad k=1,2,3, \ldots
$$

Proof. (i) By Theorem 2, $\widetilde{G}$ is an imbedded Lie subgroup of $I(\mathfrak{E})$. Then from Proposition 3.62 in [1], the induced action $\tilde{\mu}: \widetilde{G} \times \mathfrak{E} \rightarrow \mathfrak{E}$ is proper. On the other hand, let $F(h)=h$ for some $h \in \pi^{-1}\{x\}$ for some $x \in \mathfrak{M}$, where $F \in \widetilde{G}$. From Equation 4 ,

$$
\Phi_{f(x)}(h)=\left((f \circ \pi)(h),\left(p r_{2} \circ \Phi_{x}\right)(h)\right),
$$

which implies

$$
\pi(h)=\pi(F(h))=\left(\pi \circ \Phi_{f(x)}^{-1}\right)\left((f \circ \pi)(h),\left(p r_{2} \circ \Phi\right)(h)\right)=p r_{1}\left((f \circ \pi)(h),\left(p r_{2} \circ \Phi\right)(h)\right)=(f \circ \pi)(h) .
$$

Since $\mu$ is a free action, then $f=i d_{I(\mathfrak{M})}$. Because $\Omega$ is a homomorphism, then it maps identity of $I(\mathfrak{M})$ to identity of $I(\mathfrak{E})$, which implies that $F=i d_{\widetilde{G}}$. Since for any $h \in \mathfrak{E}$, and $F \in \widetilde{G}, F(h)=h$ implies $F=i d$, then $\tilde{\mu}$ is a free action.

(ii) We recall that Mann [8] has proven that if an $n$-dimensional Riemannian manifold $\mathfrak{M}$ with $n \neq 4,6,10$, then $I(\mathfrak{M})$ contains no closed subgroup $G$ where dimension of $G$ falls into any of the ranges in Equation 7. Since $\left(\mathfrak{E}, \mathfrak{g}_{\mathfrak{E}}\right)$ is a Riemannian manifold (endowed with the induced metric $\mathfrak{g}_{\mathfrak{E}}$ ), then by the Theorem $2, \widetilde{G}$ is a closed subgroup of $I(\mathfrak{E})$. Thus one can use the Mann's theorem if $\operatorname{dim}(\mathfrak{E}) \neq 4,6,10$. On the other hand, it follows from Corollary 1 that $\operatorname{Dim}(I(\mathfrak{M}))=\operatorname{Dim}(\widetilde{G})$ which shows that the dimension of $I(\mathfrak{M})$ does not fall into the ranges in Equation 7.

Proposition 1. Let $\mathfrak{M}$ be an $n$-dimensional Riemannian manifold, where $n \geq 4$. If $\mathfrak{M}$ is not of constant curvature, then

$$
\operatorname{Dim}(I(\mathfrak{M})) \leq \frac{n(n-1)}{2}+1 .
$$

Proof. Suppose that $\operatorname{Dim}(I(\mathfrak{M}))=N$. Since $n \geq 4$, then all vector bundles based on $\mathfrak{M}$ is of dimension $\operatorname{Dim}(\mathfrak{E}) \neq 4$. By Theorem 2, $\widetilde{G}$ is an $N$ - dimensional closed subgroup of $I(\mathfrak{E})$. It follows from Theorem 3.2 in [6] that the dimension of $r$-dimensional closed subgroups of any isometry group of an $n$-dimensional manifold does not fall into the range

$$
\frac{n(n-1)}{2}+1<r<\frac{n(n+1)}{2}
$$

if $n \neq 4$. Therefore $\frac{n(n-1)}{2}+1<\operatorname{Dim}(\widetilde{G})<\frac{n(n+1)}{2}$. By Corollary $1, \operatorname{Dim}(\widetilde{G})=N$ which proves that $N$ does not fall into the ranges $\frac{n(n-1)}{2}+1<N<\frac{n(n+1)}{2}$. On the other hand, if $\mathfrak{M}$ is not of constant curvature, then from Proposition 23.4 in [9], $N \neq \frac{n(n+1)}{2}$. Since $N<\frac{n(n+1)}{2}$, then Equation 9 holds.

Corollary 2. If $\mathfrak{M}$ is isometric to any of the element of $\mathfrak{A}=\left\{S^{n}, \mathbb{R}^{n}, P_{n}(\mathbb{R}), \mathbb{H}^{n}\right\}$, where $P_{n}(\mathbb{R})$ and $\mathbb{H}^{n}$ denotes $n$-dimensional projective space and n-dimensional simply connected hyperbolic space respectively, then the followings hold. 
(i) Suppose that $\mathfrak{M}$ is a Riemannian Manifold of constant curvature.There is no free canonical action on $\mathfrak{M}$ if $n \neq 1$.

(ii) If there exists an $(n+k)$-dimensional vector bundle on $\mathfrak{M}$, and the canonical action is free, then $n \in\{1,2, \ldots, t\}$ where $k=\frac{t(t-1)}{2}, t=2,3, \ldots$.

Proof. (i) Suppose that $\mathfrak{M}$ is a Riemannian manifold with constant curvature and isometric to one of the elements in $\mathfrak{A}$. It follows from Theorem 3.1 in [6] that $\operatorname{Dim}(I(\mathfrak{M}))=\frac{n(n+1)}{2}$. By Theorem 4, $\mathfrak{E} / \widetilde{G}$ is a smooth manifold of $\operatorname{dimension} \operatorname{Dim}(\mathfrak{E})-\operatorname{Dim}(\widetilde{G})$. Suppose that $\mathfrak{E}$ is a line bundle on $\mathfrak{M}$. Then $\mathfrak{E}$ is $(n+1)-$ dimensional vector bundle. Therefore $n+1>\frac{n(n+1)}{2}$ which implies $n<2$.

(ii) Suppose that there exists an $(n+k)$-dimensional vector bundle on $\mathfrak{M}$. If the canonical action is free, then the induced $\widetilde{G}$-action on $\mathfrak{E}$ is free. Moreover, since $\widetilde{G}$ is closed, then the induced action is proper. Therefore $\mathfrak{E} / \widetilde{G}$ is a smooth manifold of dimension $\operatorname{Dim}(\mathfrak{E})-\operatorname{Dim}(\widetilde{G})$ which concludes that $n+k>\frac{n(n+1)}{2}$. Thus $n^{2}+n-2 k<0$. Solving this inequality leads

$$
0<n<\frac{1+\sqrt{8 k+1}}{2}
$$

$\frac{1+\sqrt{8 k+1}}{2}$ is a positive integer if there exists a positive number $t \in \mathbb{Z}^{+}-\{1\}$ such that $2 t=1+\sqrt{8 k+1}$. Thus $k=\frac{t(t-1)}{2}$. This finishes the proof.

\section{Competing interests}

The authors declare that they have no competing interests.

\section{Authors' contributions}

All authors have contributed to all parts of the article. All authors read and approved the final manuscript.

\section{References}

[1] Alexandrino, Marcos M., Bettiol, Renato G. Lie Groups and Geometric Aspects of Isometric Actions ,Springer International Publishing Switzerland, (2015).

[2] Fox, Ralph H., On topologies for function spaces, Bull. Amer. Math. Soc., 51 , 429-432, (1945).

[3] Ihrig, Edwin., The Size of Isometry Groups on Metric Spaces, J. Mathematical Analysis and Applications, 96, 447-453, (1983).

[4] Kadioglu H., Fisher R., Metric Structures on Fibered Manifolds Through Partitions of Unity, New Trends in Mathematical Sciences, 4(2), 266-272, (2016).

[5] Kadioglu H., Prolongations of Isometric Actions to Vector Bundles, Under Review, (2016).

[6] Kobayashi, S., Transformation groups in differential geometry, Ergeb. der Math. and ihrer Grenzgeb., (70,) Springer, Berlin (1972).

[7] Myers S. B. and Steenrod N.E., The Group of Isometries of a Riemannian Manifold, The Annals of Mathematics, 40, 2, 400-416, (1939).

[8] Mann, L. N., Gaps in Dimensions of Isometry Groups of Riemannian Manifolds, J. Differential Geometry, 11, 293-298, (1976).

[9] Postnikov, M. M., Geometry VI: Riemannian Geometry, Springer Science and Business Media, (2001).

[10] Wakakuwa, H., On n-dimensional Riemannian Spaces admitting some groups of Motions of order less than n(n-1)/2, Tohoku Math. J., 2(6), 121-134, (1954).

[11] Wang, H. C., Finsler Spaces with Completely Integrable Equations of Killing, J. London Math Soc., 22, 5-9, (1947). 